\title{
Programas de exercício físico para pessoas pré- hipertensas: revisão com base no modelo RE-AIM
}

\author{
Physical exercise programs for pre-hypertensive \\ individuals: review based on the RE-AIM model
}

\author{
Camila Fabiana Rossi Squarcini, Andreza Nascimento da Silva, Clovis Lemos Bastos \\ Junior, Danielle de Amaral Macedo, Joslei Viana de Souza
}

\section{Como citar este artigo:}

SQUARCINI, Camila Fabiana Rossi;

SILVA, A. N.; BASTOS JUNIOR, C.

L.; MACEDO, D. A.; SOUZA, J. V.;

Programas de exercício físico para pessoas pré-hipertensas: revisão com base no modelo REAlM. Revista Saúde (Sta. Maria). 2019; 45 (2).

\section{Autor correspondente:}

Nome: Camila Fabiana Rossi

Squarcini

E-mail: cfrsquarcini@uesc.br

Telefone: +55 (73) 36805116

Formação Profissional: Doutora

em Educação Física pela

Universidade Federal de Santa

Catarina (UFSC), Florianópolis, SC, Brasil.

Filiação Institucional: Universidade Estadual de Santa Cruz (UESC)

Endereço para correspondência: Rodovia Jorge Amado, Km 16

Bairro: Salobrinho

Cidade: Ithéus Estado: Bahia

CEP: 45662-900

Data de Submissão:

28/02/2019

Data de aceite:

26/07/2019

Conflito de Interesse: Não há conflito de interesse

\section{(cc) $\mathrm{BY}-\mathrm{NC}-\mathrm{ND}$}

\section{RESUMO}

Há evidências em estudos com alto controle de variáveis e grupos homogêneos (validade interna) de que o exercício físico reduz os índices pressóricos em pessoas com pré-hipertensão. Entretanto, não se sabe ao certo se esses estudos apresentam informações suficientes para que tais exercícios sejam aplicados na prática clínica, no dia-a-dia (validade externa). Objetivo: avaliar se os artigos científicos sobre programas de exercício físico para pessoas com pré-hipertensão da principal base de dados biomédica apresentam informações suficientes sobre a sua validade interna e externa. Métodos: foi realizada uma revisão sistemática no PubMed entre 2007 a 2017, de artigos publicados em inglês, espanhol ou português. Para seleção dos artigos, dois pareceristas (e um terceiro em caso de discordância) avaliaram o título, o resumo e o texto completo. Após esta etapa, foi realizada a avaliação da validade interna e externa utilizando o check-list do modelo de avaliação RE-AIM. Resultados: foram encontrados 11.693 artigos para leitura dos títulos. Destes, 7 foram selecionados para leitura dos resumos, 5 artigos para leitura na íntegra, finalizando o processo com 3 artigos selecionados para a análise pelo modelo RE-AIM. Dos artigos selecionados, observou-se que atenderam apenas $33,33 \%$ dos itens das dimensões Alcance (público alvo e participante da pesquisa) e Implementação. No check-list da dimensão Efetividade/Eficácia (resultados) apenas 12,5\% foram atendidos. As dimensões Adoção (organização e equipe alvo e participante) e Manutenção não foram contempladas. Conclusão: os artigos apresentam poucas informações a respeito da validade externa, dificultando assim a possibilidade de disseminação desses protocolos de exercício físico para outras realidades.

PALAVRAS-CHAVE: Pré-Hipertensão Arterial. Atividade Física. Programa. Avaliação de Programa

\section{ABSTRACT}

There is evidence in studies with high control of variables and homogeneous groups (internal validity) that physical exercise reduces blood pressure rates in people with prehypertension. However, it is not clear whether these studies present enough information for the application of physical exercise in clinical practice, on a day-to-day basis (external validity). Aim: The objective was to evaluate if the scientific articles on physical exercise programs for people with pre-hypertension from the main biomedical database show sufficient information about internal and external validity. Methods: A systematic review was conducted in PubMed between 2007 and 2017, of articles published in English, Spanish or Portuguese. In order to select the articles, two referees (and a third in case of disagreement) evaluated the title, the abstract and the complete text. After this stage, the internal and external validity evaluation was performed using the checklist of the RE-AIM evaluation model. Results: There were 11,693 articles for reading the titles. Of these, 7 were selected for reading the abstracts, 5 articles for reading in full, finalizing the process with 3 articles selected for analysis by the RE-AIM model. Of 
the selected articles, it was observed that the articles only answered $33.33 \%$ of the items from the dimensions Reach (target population and research participant) and Implantation. In the checklist of the Effectiveness/Efficacy dimension (results) only 12.5\% were addressed. The Adoption (organization, target, participant team) and Maintenance dimensions were not considered. Conclusion: the articles present limited information about the external validity, making it difficult to disseminate these physical exercise protocols into other realities.

KEYWORDS: Pre-Hypetension. Physical Activity. Program. Program Evaluation

\section{INTRODUÇÃO}

A pré-hipertensão tem ganho espaço na saúde pública, pois é um fator de risco que ao ser cuidado, evita 0 acometimento da hipertensão arterial sistêmica ${ }^{1}$ (esta é considerada uma das cinco prioridades das agências internacionais e de diversos países no combate às doenças crônicas não transmissíveis (DCNT)2 decorrente da alta prevalência ${ }^{3}$, dificuldade de controle ${ }^{3}$, dificuldade de conscientização ${ }^{3}$, preditor para outros agravos de saúde ${ }^{4,6}$ e alto custo ${ }^{7}$.

Partindo dos parâmetros de normalidade $(<120 \mathrm{mmHg}$ para a pressão arterial sistólica e $<80 \mathrm{mmHg}$ para a pressão arterial diastólica), a pré-hipertensão é classificada quando os valores pressóricos variam entre 120 a $139 \mathrm{mmHg}$ para a pressão arterial sistólica (PAS) e entre 80 a 89 mmHg para a pressão arterial diastólica (PAD), indicando, assim o limite entre ter ou não HAS. ${ }^{1,5}$

A prevalência da pré-hipertensão varia entre $25 \%$ a $50 \%$ em todo o mundo ${ }^{8}$, sendo observado no Brasil um valor de $30,7 \%$ em $2009^{9}$. Com isso, vem sendo recomendada para as pessoas com pré-hipertensão a mudança no estilo de vida, pois esta ação tem sido capaz de reduzir em $20 \%$ o risco do desenvolvimento da $\mathrm{HAS}^{8}$ e para as pessoas com pré-hipertensão que não apresentam doença renal crônica essa mudança não precisa, necessariamente, estar acompanhada do uso de fármacos ${ }^{4}$.

Uma das mudanças de estilo de vida está relacionada à prática de atividade física, no qual tem sido indicado a prática de exercício físico com no mínimo 30 minutos em intensidade moderada, de preferência sete dias na semana ${ }^{10}$. Isto porque há comprovação que a atividade física, associada ou não com a dieta alimentar, é capaz de reduzir os índices pressóricos para as pessoas com pré-hipertensão que apresentam sobrepeso/obesidade. É observado também uma redução de 4,0 mmHg para a PAS e 2,8 mmHg para a PAD para os que praticam exercício físico durante 6 meses ${ }^{11}$.

Embora a pré-hipertensão seja um fator importante a ser considerado para a saúde pública, percebeu-se que poucos são os estudos direcionado aos programas de exercício físico e que não existe uma revisão sistemática sobre o assunto que leve em consideração itens importantes a serem considerados na prática clínica do dia-a-dia. 
Neste caso, objetivando a aplicação dos programas na prática clínica, ou seja, no dia-a-dia, o modelo de avaliação Reach (Alcance), Efficacy (Efetividade/Eficácia), Adoption (Adoção), Implementation (Implementação), Maintanance (Manutenção) - RE-AIM, previamente validada ${ }^{12,14}$ vem apresentar indicativos importantes para serem avaliados a fim de facilitar a implementação ou disseminação de um programa, no presente caso, de exercício físico.

O RE-AIM foi desenvolvido para avaliar intervenções em saúde pública, levando em considerações não apenas o resultado encontrado como também outras variáveis como: o perfil e taxa de participação dos participantes, perfil e taxa de participação organizacional e da equipe executora, taxa de abandono, manutenção do programa, mudanças necessárias para implementação, dentre outras. ${ }^{13,15} \mathrm{O}$ RE-AIM também tem sido utilizado para avaliar revisões sistemáticas no qual avalia a dimensão em que os artigos atendem ou negligenciam informações contidas nas dimensões do RE-AIM ${ }^{15}$.

Assim, o presente estudo teve como objetivo avaliar os artigos científicos que apresentam programas de exercício físico para pessoas com pré-hipertensão utilizando como base o modelo RE-AIM de avaliação.

\section{MATERIAL E MÉTODO}

Trata-se de um estudo de revisão sistemática sobre a investigação de programas de exercício físico desenvolvidos para atender pessoas com pré-hipertensão que foram publicados em artigos científicos presentes na base de dados PubMed durante o período de 2007 a 2017.

Para determinação das entradas foi realizada consultas no Medical Subject Headings (MeSH) sendo estabelecida a seguinte entrada: Prehypertension OR Pre-Hypertension OR Pre-Hypertensions OR Prehypertensions AND Physical Activity OR Physical Exercise AND Program OR Programs.

Como critérios de inclusão foram estabelecidos: a) estudos que envolviam adultos (18 ou mais anos de idade) com pré-hipertensão; b) estudos publicados entre janeiro de 2007 a dezembro de 2017; c) estudos publicados em português, inglês e espanhol; d) estudos envolvendo exercício físico.

Foram excluídos os artigos relacionados com: a) revisão da literatura, revisão sistemática ou metanálise, b) gestantes, c) animais, d) duplicidade e e) temas que não diziam respeito a pré-hipertensão. Os artigos excluídos foram contabilizados, levando em consideração os motivos para tais.

A seleção foi iniciada pela leitura dos títulos, seguindo pela leitura do resumo e do texto na íntegra. Cada etapa foi analisada por dois avaliadores e, quando existiu discordância, um terceiro avaliador fez a análise das discordâncias.

Após seleção dos artigos, foi realizada uma análise descritiva a fim de apresentar informações como: país onde o estudo foi desenvolvido, revista em que foi publicado, objetivo do estudo, tipo do estudo, número de participantes, perfil dos participantes e protocolo do exercício físico. 
Após esta etapa foi iniciada a avaliação conforme modelo RE-AIM ${ }^{12,13}$, no qual as 5 dimensões foram consideradas fundamentais para a implementação de um programa no dia-a-dia. Este modelo foi publicado em artigo por Glasgow, Vogt e Bole ${ }^{15}$ pela primeira no qual destaca que o foco concentra-se na análise da validade interna (a capacidade de controle e homogeneidade de um estudo, característicos dos estudos quantitativos randomizados) e da validade externa (a capacidade de um estudo poder ser generalizado, ou seja, aplicado na vida real, com populações e situações diferentes) do estudo. Para tanto, foi utilizado o check-list para revisões da literatura do próprio modelo ${ }^{16}$.

Assim, avaliou-se:

Dimensão Alcance: População Alvo, Perfil da População Alvo, Método de Identificação da População Alvo, Estratégia de Recrutamento, Critério Inclusão, Critério Exclusão, Número Elegíveis da População Exposta ao Recrutamento, Tamanho da Amostra, Taxa de Participação, Comparação Estatística - população alvo e participantes, Custo do Recrutamento e Uso de Metodologia Qualitativa.

Dimensão Efetividade/Eficácia: Mediadores (variáveis que causam um resultado, diferente das variáveis moderadoras), Intensão de Tratamento, Procedimentos de Imputação dos Dados, Mensuração de Qualidade de Vida, Resultados ou Consequências Negativas, Custo da Efetividade e Uso de Metodologia Qualitativa.

Dimensão Adoção: em seu Nível Organizacional: Elegibilidade Organizacional, Número de Organizações Alvo, Taxa de Participação Organizacional, Descrição Organização Alvo, Critério Inclusão/ Exclusão Organizacional, Descrição do Local de Intervenção, Método para Identificar a Organização Participante, Comparação Organizações Participantes e não Participantes, Análise Estatística Comparando as Organizações e Número de Pessoas da Equipe Servidas. Em seu nível individual: Número de Membros da Equipe Elegíveis e Convidados, Número de Membros da Equipe Participantes, Taxa de Participação, Método para Identificar a Equipe Participante, Nível de Formação da Equipe, Critério Inclusão/ Exclusão da Equipe, Comparação entre Equipe Participante e não Participante, Análise Estatística Comparando as Equipes, Custo do Recrutamento, Disseminação além do Planejado, Uso de Metodologia Qualitativa

Dimensão Implementação: Apresenta Teoria, Número de Intervenções, Tempo de Contato, Duração do Contato, Entrega do Protocolo como Planejado, Consistência da Implementação, Taxa de Participação versus Conclusão, Custo e Uso de Metodologia Qualitativa.

Dimensão Manutenção: Nível organizacional: Reportou Coerência com a Missão Organizacional, O Programa ainda Ocorre na Organização, Motivos para o Término do Programa, O Programa foi Institucionalizado, Qts Lugares e Motivos de Parar com o Programa e Uso de Método Qualitativo. Nível Individual: Os Participantes Mantiveram as Ações do Programa, Qts Participantes e Seus Motivos de Parar com o Programa e Uso de Metodologia Qualitativa. 
Para a análise foi utilizada a análise descritiva (frequência absoluta e relativa) por meio do programa estatística Statistical Package for the Social Sciences, versão 16.0®. O fluxograma seguiu um modelo adaptado do PRISMA ${ }^{17}$, a fim de contabilizar os artigos selecionados e retirados, levando em consideração os motivos para tais retiradas.

\section{RESULTADOS}

Após a busca na base de dados do PubMed foram encontrados 11.693 títulos, dos quais 7 permaneceram após a leitura do título, 5 após a leitura do resumo e 3 selecionados para a leitura na íntegra. Dentre os principais motivos de exclusão dos artigos estavam: falta de relacionamento com tema abordado, estudo de revisão e estudo com gestantes (ver Figura 1).

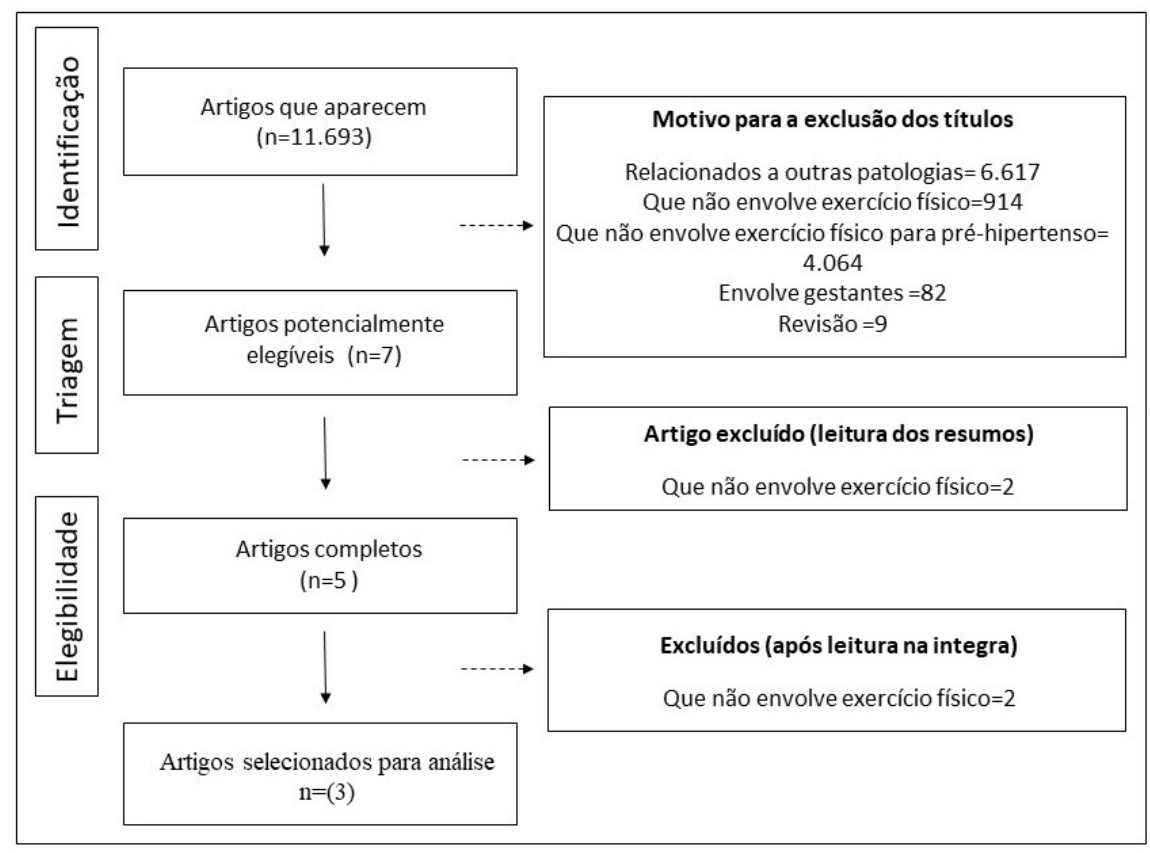

Figura 1. Fluxograma do processo de seleção de artigos sobre programas de atividade física elou exercício físico para pessoas com pré-hipertensão arterial na base de dados Pubmed adaptado de PRISMA ${ }^{17}$.

Os três artigos selecionados e avaliados foram de países da América e utilizaram a abordagem quantitativa como forma para investigar o efeito do exercício físico (associado ou não com a alimentação) em participantes de ambos os sexos (exceto o estudo de Sales et al., 2012, que investigaram apenas as mulheres) - ver Quadro 1.

Quadro 1. Perfil geral dos estudos sobre programas de atividade física elou exercício físico para pessoas com préhipertensão arterial publicados no PubMed entre 2007-2017. Ilhéus- BA. 2018. 


\begin{tabular}{|c|c|c|c|}
\hline & Sales et al. $(2012)^{18}$ & Beck et al. (2013) ${ }^{19}$ & $\begin{array}{l}\text { Márquez-Celedonio et al. } \\
(2009)^{20}\end{array}$ \\
\hline País & Brasil & EUA & México \\
\hline Revista & $\begin{array}{l}\text { European Journal of } \\
\text { Applied Physiology }\end{array}$ & $\begin{array}{c}\text { Experimental Biology and } \\
\text { Medicine }\end{array}$ & $\begin{array}{c}\text { Revista Española de } \\
\text { Cardiología }\end{array}$ \\
\hline Objetivo & $\begin{array}{l}\text { Investigar o efeito da } \\
\text { dieta e exercício físico } \\
\text { na pressão arterial e } \\
\text { modulação autonômica } \\
\text { em mulheres com pré- } \\
\text { hipertensão. }\end{array}$ & $\begin{array}{c}\text { Examinar o efeito do } \\
\text { treinamento resistido e de } \\
\text { endurance separadamente } \\
\text { na função da condução da } \\
\text { artéria endotelial em jovens } \\
\text { pré-hipertensos }\end{array}$ & $\begin{array}{l}\text { Determinar o efeito da } \\
\text { mudança no estilo de } \\
\text { vida clínica no risco } \\
\text { cardiovascular de } \\
\text { indivíduos pré-hipertensos }\end{array}$ \\
\hline Tipo Estudo & $\begin{array}{l}\text { Quantitativo, não } \\
\text { randomizado }\end{array}$ & Quantitativo, randomizado & $\begin{array}{l}\text { Quantitativo, } \\
\text { randomizado }\end{array}$ \\
\hline Número de participantes & 20 & 58 & 92 \\
\hline Perfil dos Participantes & $\begin{array}{c}\text { Mulheres adultas, pré- } \\
\text { hipertensas e normotensas }\end{array}$ & $\begin{array}{l}\text { Jovens, ambos sexos, pré- } \\
\text { hipertensos e normotensos }\end{array}$ & $\begin{array}{c}\text { Adultos, ambos sexos, pré- } \\
\text { hipertensos }\end{array}$ \\
\hline Protocolo de treinamento & $\begin{array}{c}\text { Exercício físico aeróbio } \\
\text { e resistido, } 12 \text { horas } \\
\text { semanas, com } 3 \text { sessões } \\
\text { por semana de } 70 \text { minutos } \\
\text { cada }\end{array}$ & $\begin{array}{c}\text { Exercício físico resistido } \\
\text { ou exercício de endurance. } \\
3 \text { sessões diárias de } \\
60 \text { minutos durante } 8 \\
\text { semanas. }\end{array}$ & $\begin{array}{l}\text { Exercício físico durante } 3 \\
\text { a } 5 \text { sessões por semana } \\
\text { (exercício aeróbico e } \\
\text { sessões de esportes com } \\
\text { duração de } 45 \text { minutos } \\
\text { cada. }\end{array}$ \\
\hline
\end{tabular}

No que se refere a dimensão Alcance do modelo RE-AIM (Quadro 2), apenas 33,3\% dos indicadores foram apresentados nos artigos. Neste caso, os itens citados por todos foram os critérios de inclusão e o tamanho da amostra. A estratégia de recrutamento foi um item citado apenas em um artigo, assim como ocorreu para a apresentação dos critérios de exclusão.

Nesse sentido, permanece obscuro do ponto de vista de uma possível replicação, ou seja, disseminação desse programa de exercício físico para outras pessoas com pré-hipertensão uma vez que importantes informações a respeito da população não foram apresentadas. Informações como: quantos são, qual o perfil deles, quantos foram convidados para participarem do estudo, qual a taxa de participação, qual critério utilizado para convidar os participantes, qual a diferença entre quem participou e quem não aceitou participar do estudo, qual o custo com essa etapa, deveriam ser descritas nos artigos. Além disso, também não foi relatado o uso de técnica qualitativa para esta etapa.

Quadro 2. Indicadores da dimensão Alcance do modelo RE-AIM dos estudos sobre programas de atividade física e/ou exercício físico para pessoas com pré-hipertensão arterial. Ilhéus- BA. 2018. 


\begin{tabular}{|c|c|c|c|c|}
\hline Alcance & $\begin{array}{c}\text { Sales et al. } \\
(\mathbf{2 0 1 2})^{18}\end{array}$ & $\begin{array}{c}\text { Beck et al. } \\
(\mathbf{2 0 1 3})^{19}\end{array}$ & $\begin{array}{c}\text { Márquez- } \\
\text { Celedonio } \\
\text { et al. (2009) })^{20}\end{array}$ & Total \\
\hline População Alvo & - & - & - & $0 \%$ \\
\hline Perfil da População Alvo & - & - & - & $0 \%$ \\
\hline Método de Identificação da População Alvo & - & - & - & $0 \%$ \\
\hline Estratégia de Recrutamento & Sim & - & - & $33 \%$ \\
\hline Critério Inclusão & Sim & Sim & Sim & $100 \%$ \\
\hline Critério Exclusão & - & - & Sim & $33 \%$ \\
\hline Número Elegíveis da População Exposta ao \\
Recrutamento & - & - & - & $0 \%$ \\
\hline Tamanho da Amostra & - & Sim & Sim & $100 \%$ \\
\hline Taxa de Participação & - & - & - & $0 \%$ \\
\hline Comparação Estatística - população alvo e \\
participantes
\end{tabular}

Quadro 3. Indicadores da dimensão Efetividade/Eficácia do modelo RE-AIM dos estudos sobre programas de atividade física e/ou exercício físico para pessoas com pré-hipertensão arterial. Ilhéus- BA. 2018.

\begin{tabular}{|c|c|c|c|c|}
\hline Efetividade & $\begin{array}{c}\text { Sales et al. } \\
\mathbf{( 2 0 1 2}^{\mathbf{1 8}}\end{array}$ & $\begin{array}{c}\text { Beck et al. } \\
\mathbf{( 2 0 1 3}^{\mathbf{1 9}}\end{array}$ & $\begin{array}{c}\text { Márquez- } \\
\text { Celedonio } \\
\text { et al. (2009) }\end{array}$ & $\begin{array}{c}\text { Total } \\
\text { Mediadores* }\end{array}$ \\
\hline Intensão de Tratamento & - & Sim & Sim & $100 \%$ \\
\hline Procedimentos de Imputação dos Dados & - & - & - & $0 \%$ \\
\hline Mensuração de Qualidade de Vida & - & - & - & $0 \%$ \\
\hline Resultados ou Consequências Negativas & - & - & - & $0 \%$ \\
\hline Custo da Efetividade & - & - & - & $0 \%$ \\
\hline Uso de Metodologia Qualitativa & - & - & - & $0 \%$ \\
\hline
\end{tabular}

* mediadores - são aquelas variáveis preditoras de um resultado (causa um efeito)

Sobre a dimensão Efetividade/ Eficácia do modelo RE-AIM (Quadro 3), os artigos apresentaram as variáveis dependentes e independentes. Entretanto, as demais informações a respeito dessa dimensão não são informadas. Informações estas que são importantes para compreender quantas pessoas abandonaram o processo durante a execução do programa ("intensão de tratamento"), se as abstenções no programa foram levadas em consideração ("procedimentos de imputação de dados"), se a qualidade de vida foi uma variável considerada, assim como, se os resultados adversos 
ocorreram e quais poderiam ter sido eles, usualmente relatado em uma abordagem mais profunda, caso da metodologia qualitativa.

Sobre a dimensão Adoção do modelo RE-AIM (Quadro 4), fica nítido que esta não é uma variável levada em consideração durante a produção de um artigo científico, independentemente se o nível é organizacional ou individual.

No que se refere à dimensão Implementação, conforme check-list do modelo RE-AIM (ver Quadro 5), embora sejam apresentadas o número de intervenções, o tempo de contato e a duração dos programas, as demais informações não foram mencionadas nos artigos. Por fim, no que se refere à dimensão Manutenção nenhuma informação foi apresentada nos artigos, em nível organizacional ou individual (Quadro 6).

Quadro 4. Indicadores da dimensão Adoção do modelo RE-AIM dos estudos sobre programas de atividade física e/ou exercício físico para pessoas com pré-hipertensão arterial. Ilhéus- BA. 2018.

\begin{tabular}{|c|c|c|c|c|}
\hline Adoção & $\begin{array}{l}\text { Sales et al. } \\
(2012)^{18}\end{array}$ & $\begin{array}{l}\text { Beck et al. } \\
(2013)^{19}\end{array}$ & $\begin{array}{c}\text { Márquez- } \\
\text { Celedonio } \\
\text { et al. }(2009)^{20}\end{array}$ & Total \\
\hline \multicolumn{5}{|l|}{ Nível Organizacional } \\
\hline Elegibilidade Organizacional & - & - & - & $0 \%$ \\
\hline Número de Organizações Alvo & - & - & - & $0 \%$ \\
\hline Taxa de Participação Organizacional & - & - & - & $0 \%$ \\
\hline Descrição Organização Alvo & - & - & - & $0 \%$ \\
\hline Critério Inclusão/ Exclusão Organizacional & - & - & - & $0 \%$ \\
\hline Descrição do Local de Intervenção & - & - & - & $0 \%$ \\
\hline Método para Identificar a Organização Participante & - & - & - & $0 \%$ \\
\hline $\begin{array}{c}\text { Comparação Organizações Participantes e não } \\
\text { Participantes }\end{array}$ & - & - & - & $0 \%$ \\
\hline Análise Estatística Comparando as Organizações & - & - & - & $0 \%$ \\
\hline Número de Pessoas da Equipe Servidas & - & - & - & $0 \%$ \\
\hline \multicolumn{5}{|l|}{ Nível Individual } \\
\hline $\begin{array}{l}\text { Número de Membros da Equipe Elegíveis e } \\
\text { Convidados }\end{array}$ & - & - & - & $0 \%$ \\
\hline Número de Membros da Equipe Participantes & - & - & - & $0 \%$ \\
\hline Taxa de Participação & - & - & - & $0 \%$ \\
\hline Método para Identificar a Equipe Participante & - & - & - & $0 \%$ \\
\hline Nível de Formação da Equipe & - & - & - & $0 \%$ \\
\hline Critério Inclusão/ Exclusão da Equipe & - & - & - & $0 \%$ \\
\hline $\begin{array}{c}\text { Comparação entre Equipe Participante e não } \\
\text { Participante }\end{array}$ & - & - & - & $0 \%$ \\
\hline Análise Estatística Comparando as Equipes & - & - & - & $0 \%$ \\
\hline Custo do Recrutamento & - & - & - & $0 \%$ \\
\hline
\end{tabular}




\begin{tabular}{|c|c|c|c|c|}
\hline Disseminação além do Planejado & - & - & - & $0 \%$ \\
\hline Uso de Metodologia Qualitativa & - & - & - & $0 \%$ \\
\hline
\end{tabular}

Quadro 5. Indicadores da dimensão Implementação do modelo RE-AIM dos estudos sobre programas de atividade física e/ou exercício físico para pessoas com pré-hipertensão arterial. Ilhéus- BA. 2018.

\begin{tabular}{|c|c|c|c|c|}
\hline Implementação & $\begin{array}{l}\text { Sales et al. } \\
(2012)^{18}\end{array}$ & $\begin{array}{c}\text { Beck et al. } \\
(2013)^{19}\end{array}$ & $\begin{array}{c}\text { Márquez- } \\
\text { Celedonio } \\
\text { et al. }(2009)^{20}\end{array}$ & Total \\
\hline Apresenta Teoria & - & - & - & $0 \%$ \\
\hline Número de Intervenções & Sim & Sim & Sim & $100 \%$ \\
\hline Tempo de Contato & Sim & Sim & Sim & $100 \%$ \\
\hline Duração do Contato & $\operatorname{Sim}$ & Sim & Sim & $100 \%$ \\
\hline Entrega do Protocolo como Planejado & - & - & - & $0 \%$ \\
\hline Consistência da Implementação & - & - & - & $0 \%$ \\
\hline Taxa de Participação versus Conclusão & - & - & - & $0 \%$ \\
\hline Custo & - & - & - & $0 \%$ \\
\hline Uso de Metodologia Qualitativa & - & - & - & $0 \%$ \\
\hline
\end{tabular}

Quadro 6. Indicadores da dimensão Manutenção do modelo RE-AIM dos estudos sobre programas de atividade física e/ ou exercício físico para pessoas com pré-hipertensão arterial. Ilhéus- BA. 2018.

\begin{tabular}{|c|c|c|c|c|}
\hline Adoção & $\begin{array}{l}\text { Sales et al. } \\
(2012)^{18}\end{array}$ & $\begin{array}{l}\text { Beck et al. } \\
(2013)^{19}\end{array}$ & $\begin{array}{c}\text { Márquez- } \\
\text { Celedonio } \\
\text { et al. }(2009)^{20}\end{array}$ & Total \\
\hline \multicolumn{5}{|l|}{ Nível Organizacional } \\
\hline Reportou Coerência com a Missão Organizacional & - & - & - & $0 \%$ \\
\hline O Programa ainda Ocorre na Organização & - & - & - & $0 \%$ \\
\hline Motivos para o Término do Programa & - & - & - & $0 \%$ \\
\hline O Programa foi Institucionalizado & - & - & - & $0 \%$ \\
\hline Qts Lugares e Motivos de Parar com o Programa & - & - & - & $0 \%$ \\
\hline Uso de Método Qualitativo & - & - & - & $0 \%$ \\
\hline \multicolumn{5}{|l|}{ Nível Individual } \\
\hline Os Participantes Mantiveram as Ações do Programa & - & - & - & $0 \%$ \\
\hline $\begin{array}{c}\text { Qts Participantes e Seus Motivos de Parar com o } \\
\text { Programa }\end{array}$ & - & - & - & $0 \%$ \\
\hline Uso de Metodologia Qualitativa & - & - & - & $0 \%$ \\
\hline
\end{tabular}




\section{DISCUSSÃO}

Após avaliação dos estudos relacionados a pré-hipertensão na base de dados PubMed, os poucos artigos selecionados apresentaram poucas informações que facilitam a implementação dos programas no dia-a-dia. Essa informação seria interessante na medida em que tais programas demonstraram redução pressórica pela prática do exercício físico (individualmente ou em conjunto com a mudança alimentar), conforme o indicado por Chobanian et al. ${ }^{4}$ de que alguns casos de pré-hipertensão não necessitam de intervenção farmacológica para redução pressórica.

Outra característica que chama a atenção, presente nos três estudos, é a natureza quantitativa, utilizando a randomização como fator de seleção entre os grupos (intervenção e controle) e sendo desenvolvidos dentro de laboratórios ou universidades, o que aumenta a validade interna ${ }^{15}$.

Por outro lado, ao observar os itens atendidos no check-list do modelo RE-AIM, a validade externa desses estudos fica comprometida porque foram pouco contempladas ou porque não foram citadas (a exemplo das dimensões Adoção e Manutenção). Neste caso, vale mencionar que o RE-AIM se propõe a avaliar o potencial ou o real impacto dos estudos sobre programas de intervenção no que diz respeito a sua aplicabilidade na saúde pública ${ }^{13}$.

No entanto, como avaliar seu impacto na saúde pública se informações como equipe participante do programa, custo, qualidade de vida, dentre outras informações não foram mencionadas nesses três estudos? Ressalta-se, que 0 RE-AIM é um modelo completo de avaliação, e que pode não apresentar todas suas informações em um único artigo. Entretanto, não foram encontrados outros artigos no Pubmed que daria continuidade a publicação das informações restantes.

No campo da pesquisa, mais informações poderiam ser incluídas nos artigos para facilitar essa tradução para o dia-a-dia. Neste caso, responder questões propostas por Glasgow e Estrabooks ${ }^{21}$ no formato Quem, O que, Onde, Como, Quando e Por quê, facilitaria o acesso às informações do programa.

A dificuldade de encontrar mais informações nesse estudo, também ocorreram em outros estudos. No estudo de Konrad ${ }^{22}$ sobre avaliação de programas de mudança de comportamento, 26 artigos foram avaliados e os autores relataram que apenas alguns artigos contemplam os itens de avaliação do RE-AIM e que isso pode comprometer a validade externa. A dimensão mais reportada neste estudo foi o Alcance com $61 \%$ dos itens atendidos, seguido pela Efetividade/Eficácia com 40\%, a Adoção com 17\%, a Implementação com $26 \%$ e, por último, a Manutenção com apenas $6 \%$ dos itens atendidos.

Squarcini et al. ${ }^{23}$, em seu estudo sobre programas de atividade física para idosos, com avaliação de 26 artigos, encontrou em apenas dois estudos a citação das cinco dimensões do RE-AIM, atendendo, em média, 47\% dos itens do Alcance, 37\% da Efetividade/Eficácia, 28\% da Implementação, 6\% da Adoção e 5\% da Manutenção. 
No estudo de Silva et al. ${ }^{24}$ que relatam sobre Programas de intervenção para atividade física nas escolas brasileiras, avaliando 15 artigos observou-se que o Alcance foi a dimensão mais reportada (74\%), seguido pela Efetividade/Eficácia com 48\%, Adoção com 43\%, Implementação com 35\% e Manutenção com 5\%. Ainda, segundo os autores, as informações de ordem demográfica e comportamental da população alvo, número de unidades organizacionais envolvidas, tamanho da amostra, método de identificação da população-alvo e taxa de participação foram as mais frequentes, indicando a validade interna dos estudos.

Com base nesses estudos e seus resultados, é possível verificar que o RE-AlM é uma ferramenta com muitos itens a serem avaliados e que os artigos em sua maioria não apresentam todos seus itens, principalmente os critérios apresentados na Manutenção. Vale enfatizar que o RE-AIM tem o objetivo de avaliar artigos relacionados à saúde, tendo em vista a pontuação das qualidades dos programas de saúde e as possibilidades de realização desses programas em uma sociedade com ambiente sustentável25.

A limitação do estudo está na utilização de apenas uma base de dados, o Pubmed, uma base de dados biomédica, mas que pouco aborda as questões relacionadas com a psicologia comportamental. Neste caso, esperase com a continuidade desse estudo, avaliar outras bases de dados a fim de encontrar estudos de base comunitária, aumentando assim os relatos relacionados com a validade externa.

Sobre os resultados, ao término da intervenção do estudo de Sales et al. (2012)17, foi observada redução significativa para a PAS em mulheres com pré-hipertensão. Tal redução também foi observada no estudo de Beck et al. $(2013)^{18}$, tanto para a PAS quanto para a PAD de jovens com pré-hipertensão. Redução está também observada no estudo de Márquez-Celedonio et al. (2007) ${ }^{19}$.

\section{CONSIDERAÇÕES FINAIS}

Ao realizar este estudo de revisão sistemática pôde-se concluir que os estudos publicados no PubMed entre os anos de 2007 a 2017 em relação à Programas de exercício físico para pessoas pré-hipertensas são poucos e trazem poucas informações a respeito da validade externa, dificultando assim a possibilidade de implementação desses programas em outras realidades, como, por exemplo, as Unidades Básicas de Saúde, mesmo tendo sido observado que os exercícios físicos aeróbios e resistidos empregados sejam eficazes na redução pressórica da pressão arterial das pessoas com pré-hipertensão. 


\section{AGRADECIMENTOS}

À Pró-reitora de Pesquisa da UESC, pelo financiamento da pesquisa (projeto nº 00220.1600.1834) e iniciação científica voluntária.

\section{REFERÊNCIAS}

1. National High Blood Pressure Education Program. The seventh report of the joint national committee on prevention, detection, evaluation, and treatment of high blood pressure. Bethesda: National Heart, Lung, and Blood Institute; 2004.Beaglehole R. et al. Priority actions for the non-communicable disease crisis. Lancet. 2011;377(9775):1438-47.

2. Chow CK. et al. Prevalence, Awareness, Treatment, and Control of Hypertension in Rural and Urban Communities in High-, Middle-, and Low-Income Countries. JAMA. 2013;310(9):959-68.

3. Kokkinos P. et al. Exercise capacity and mortality in hypertensive men with and without additional risk factors. Hypertension. 2009;53(3):494-9.

4. Chobanian AV. et al. Joint National Committee on Prevention, Detection, Evaluation, and Treatment of High Blood Pressure. National Heart, Lung, and Blood Institute. National High Blood Pressure Education Program Coordinating Committee: National Committee on Prevention, Detection, Evaluation, and Treatment of High Blood Pressure; 2003.

5. Vasan RS. et al. Impact of high normal blood pressure on the risk of cardiovascular disease. N Engl J Med. 2001;345(18):1291-7.

6. Dib MW, Riera R, Ferraz MB. Estimated annual cost of arterial hypertension treatment in Brazil. Rev Panam Salud Publica. 2010;27(2):125-31.

7. Egan BM. et al. Prehypertension--prevalence, health risks, and management strategies. Nat Rev Cardiol. 2015;12(5):289-300. 
8. Malachias MVB. et al. $7^{\text {a }}$ Diretriz Brasileira de Hipertensão Arterial. Arq Bras Cardiol. 2016; 107(3Supl.3):183.

9. Ghorayeb N. et al. Diretriz em cardiologia do esporte e do exercício da Sociedade Brasileira de Cardiologia e da Sociedade Brasileira de Medicina do Esporte. Arq Bras Cardiol. 2013;100(1Supl.2):1-41.

10. Neves M, Oigman WA. Pré-hipertensão: uma visão contra o tratamento medicamentoso. Rev Bras Hipertens. 2009;16(2):112-5.

11. RE-AIM. Question: What is RE-AIM? Disponível em <http://www.re-aim.org/about/frequently-asked-questions/>. Acesso em: 21 fev. 2019.

12. Almeida FA, Brito FA, Estabrooks PA. Modelo RE-AIM: Tradução e adaptação cultural para o Brasil. REFACS (online). 2013;1(1):6-16.

13. Akers JD, Estabrooks PA, Davy BM. Translational research: bridging the gap between long-term weight loss maintenance research and practice. J Am Diet Assoc. 2010;110(10):1511-22.

14. Glasgow RE, Vogt TM, Boles SM. Evaluating the public health impact of health promotion interventions: the RE-AIM framework. Am J Public Health. 1999;89(9):1322-7.

15. RE-AIM. Tools \& resources. Disponível em <http://www.re-aim.org/wp-content/uploads/2016/09/literaturecoding.pdf>. Acesso em: 21 fev. 2019.

16. SALES, A. R. et al. Diet and exercise reduce blood pressure and improve autonomic modulation in women with pre-hypertension. Eur J Appl Physiol. 2012;112(9):3369-78.

17. Moher D. et al. Preferred reporting items for systematic reviews and meta-analyses: the PRISMA statement. PLoS Med.2009;6(7):e1000097.

18. Beck DT, Casey DP, Martin JS, Braith RW. Exercise training improves endothelial function in young 
prehypertensives. Exp Biol Med. 2013;238(4):433-41.

19. Márquez-Celedonio FG, Téxon-Fernández O, Chávez-Negrete A, Hernández-López, S, Berlín-Lascurain S. Clinical effect of lifestyle modification on cardiovascular risk in prehypertensives: PREHIPER I study. Rev Esp Cardiol. 2009;62(1):86-90.

20. Glasgow RE, Estrabooks PE. Pragmatic applications of RE-AIM for health care initiatives in community and clinical settings. Disponível em <https://www.cdc.gov/pcd/issues/2018/17_0271.htm>. Acesso em: 21 fev. 2019.

21. Konrad LM. et al. Avaliação de programas de mudança de comportamento usando a ferramenta RE-AIM: um estudo de revisão sistemática. Rev Bras Ativ Fís Saúde. 2017;22(5):439-49.

22. Squarcini CFR. et al. Physical activity programs for elderly persons: an evaluation of Brazilian scientific production using the RE-AIM framework. Rev Bras Geriatr Gerontol. 2015;18(4):909-20.

23. Silva JA. et al. Programas de intervenção para atividade física nas escolas Brasileiras: Revisão com base no modelo RE-AIM. Cien Saude Colet. Disponível em: <http://www.cienciaesaudecoletiva.com.br/artigos/programas-de-intervencao-para-atividade-fisica-nas-escolas-brasileiras-revisao-com-base-no-modelo-reaim/16669?id=16669>. Acesso em: 21 fev. 2019.

24. Benedetti TRB. et al. RE-AIM: uma proposta de avaliação de programas de atividade física. Rev Kairós Gerontol. 2014;17(2):295-314. 